\title{
A!
}

This is an electronic reprint of the original article.

This reprint may differ from the original in pagination and typographic detail.

EIMossalmy, Mohammed; Han, Zhu; Pan, Miao; Jäntti, Riku; Seddik, Karim; Li, Geoffrey

\section{Backscatter Communications over Ambient OFDM Signals using Null Subcarriers}

\section{Published in:}

Proceedings of the IEEE Global Communications Conference

DOI:

10.1109/GLOCOM.2018.8647245

Published: 01/01/2018

Document Version

Peer reviewed version

Please cite the original version:

EIMossalmy, M., Han, Z., Pan, M., Jäntti, R., Seddik, K., \& Li, G. (2018). Backscatter Communications over Ambient OFDM Signals using Null Subcarriers. In Proceedings of the IEEE Global Communications Conference (IEEE Global Communications Conference). IEEE. https://doi.org/10.1109/GLOCOM.2018.8647245

This material is protected by copyright and other intellectual property rights, and duplication or sale of all or part of any of the repository collections is not permitted, except that material may be duplicated by you for your research use or educational purposes in electronic or print form. You must obtain permission for any other use. Electronic or print copies may not be offered, whether for sale or otherwise to anyone who is not an authorised user. 


\title{
Backscatter Communications over Ambient OFDM Signals using Null Subcarriers
}

\author{
Mohamed A. ElMossallamy*, Zhu Han*^, Miao Pan*, Riku Jäntti ${ }^{\dagger}$, Karim G. Seddik ${ }^{\ddagger}$ and Geoffrey Ye Li ${ }^{\S}$ \\ *Electrical and Computer Engineering Department, University of Houston, TX 77004, USA \\ ${ }^{\diamond}$ Department of Computer Science and Engineering, Kyung Hee University, Seoul, South Korea \\ ${ }^{\dagger}$ Department of Communications and Networking, Aalto University, Espoo, Finland \\ ${ }^{\ddagger}$ Electronics and Communications Engineering Department, American University in Cairo, AUC Avenue, New Cairo 11835, Egypt \\ ${ }^{\S}$ School of Electrical and Computer Engineering, Georgia Institute of Technology, Atlanta, GA, USA
}

\begin{abstract}
In recent years, ambient backscatter communications have gained a lot of interest as a promising enabling technology for Internet-of-Things and green communications. In ambient backscatter communication systems, battery-less devices are able to transmit information by backscattering ambient RF signals generated by legacy communication systems such as digital TV broadcasting, Wi-Fi, or cellular. This paper is concerned with ambient backscatter communications over legacy cellular OFDM signals. We propose a novel modulation scheme that allows backscattering devices to take advantage of the spectrum structure of ambient OFDM symbols to transmit information. We analyze the error performance of the proposed scheme, provide an exact expression for the error probability, and validate our analysis using Monte-Carlo simulation. We investigate the effects of varying the OFDM symbol size and maximum channel delay spread on the error performance. Our numerical results show that the proposed technique outperforms other techniques available in the literature for backscatter communication over ambient OFDM signals in different scenarios.
\end{abstract}

Index Terms-Ambient backscatter, internet of things, green communications, performance analysis, OFDM.

\section{INTRODUCTION}

Ambient backscatter is an intriguing new paradigm that turns ambient radio-frequency (RF) signals from a source of interference to an opportunity for communications. Traditional backscatter is a mature technology [1] that has been used for many years to achieve short-range communications in powerconstrained scenarios (e.g. RFID). In traditional backscatter communication systems, a dedicated device has to generate a continuous sinusoidal signal, which is phase-shifted and backscattered by tags by intentionally changing their antenna impedance to transmit information back to a reader device. However, in ambient backscatter, ambient RF transmission, which is vastly available (e.g. TV broadcast, cellular or Wi$\mathrm{Fi}$ ), is used instead of requiring the transmission of a dedicated sinusoidal signal. This makes ambient backscatter an attractive candidate for pervasive ultra-low power wireless networks.

The idea of ambient backscatter has been first introduced in [2], where ambient digital TV signals have been used to establish communication between two battery-less tags in

This work is partially supported by US MURI, NSF CNS-1717454, CNS1731424, CNS-1702850, CNS-1646607. a device-to-device (D2D) manner. A simple prototype has been developed to demonstrate transmission with rates up to $1 \mathrm{Kbps}$ over a somewhat modest communication range of 2.5 feet. Subsequent research [3]-[6] has significantly improved transmission rates and communication range. In [3], Internet connectivity to battery-less RF-powered devices can be provided using two off-the-shelf commercial Wi-Fi access points (APs). Uplink rates of up to $1 \mathrm{kbps}$ and a communication range of up to 2 meters have been achieved by modulating the channel state information (CSI) and received signal strength indicator (RSSI) signals in the WiFi packets, while downlink rates of up to $20 \mathrm{kbps}$ and a communication range of up to 3 meters have been reached by using a clearto-send-to-self (CTS-to-self) packet to silence other devices and then information is encoded in short WiFi packets (i.e. ' 1 ': send packet, ' 0 ': remain silent). The technique in [4] can achieve data rates of up to $1 \mathrm{Mbps}$ and a communication range of 25 meters between two battery-less devices, which is made possible by two improvements over [2]: (1) direct-link (i.e. legacy-transmitter to reader) interference cancellation, and (2) orthogonal coding similar to CDMA chip sequences. Later in [5], rates of up to $5 \mathrm{Mbps}$ and a communication range of up to 5 meters can be reached between a battery-less tag and a WiFi AP by using full-duplex radio. Moreover, it has been demonstrated in [6] that WiFi packets can be synthesized by backscattering Bluetooth Low Energy (BLE) signals.

Performance analysis of ambient backscatter communication systems can be found in [7]-[12]. In [7], it has been shown that adding backscattering nodes to a legacy MIMO communication system increases the achievable sum rate. In [8], signal detection and error performance of an ambient backscatter communication system have been investigated where the tag uses a differential encoder to exempt the reader from estimating the channel, and an averaging technique, similar to the prototype in [2], is used for detection. The same approach has been extended to the case when the reader has multiple antennas in [9]. Motivated by the fact that OFDM is the prevalent modulation scheme in most modern communications systems (e.g. DVB, LTE, WiFi), backscatter communications over ambient OFDM carrier signals has been studied in [11], [12]. In [11], by taking advantage of the 
structure of the OFDM symbol in the time-domain, a modulation scheme for the ambient backscatter system has been designed, which canceled direct-link interference using the remaining part of the cyclic prefix. Moreover, the fundamental information-theoretic limits of backscatter communication over ambient OFDM carriers, such as ergodic and outage capacity, have been investigated in [12]. It has been shown that ambient backscatter not only allows a battery-less tag to opportunistically communicate at satisfactory rates over short distances, but can also benefit legacy transmission by offering a form of diversity.

In this paper, we investigate backscatter communications over ambient OFDM signals using null subcarriers. Our contributions in this paper can be summarized as follows:

- We propose a novel modulation scheme for backscatter communications over ambient OFDM carriers. We design the tag modulation waveform and the detector to avoid direct-link interference.

- We analyze the error performance of the proposed scheme and obtain an exact expression for the error probability in terms of the bivariate Meijer G-function.

- We further study the effects of system parameters, namely, the maximum channel delay spread, and OFDM symbol size, on the error performance of the proposed modulation scheme.

The rest of the paper is organized as follows. In Section II, we present the system model. In Section III, we introduce our novel modulation scheme for backscatter communications over ambient OFDM signals and investigate the error performance of our scheme. In Section IV, we present simulation results to corroborate our analysis, compare our scheme with existing schemes in the literature, and study the effects of some system parameters on the error performance. Finally, in Section V, we conclude the paper.

\section{SySTEM MOdeL}

In this section, we present our system model. In general, we have two co-existing communications systems as in Fig. 1. A legacy communication system that employs OFDM, for example, LTE or WiFi, and a capillary ambient backscatter communication system consisting of ultralow power tags/sensor nodes and readers. The legacy nodes are not power-constrained and are either powered by largecapacity batteries (e.g. user equipment) or the power-grid (e.g. base stations), while the capillary tags/sensor nodes rely on RF-energy harvesting for power and can only communicate by rescattering the legacy system OFDM transmission. The readers can either be independent devices or part of the legacy nodes [12].

Next, we assume there are one legacy transmitter, one tag, and one reader, and they all have a single antenna each. The tag modulates its information into the ambient OFDM signal by intentionally altering its antenna impedance to phase-shift and rescatter the ambient signal so that the reader can decode the information by observing the difference in received energy. Hence, the tag does not require any power-hungry RF chains

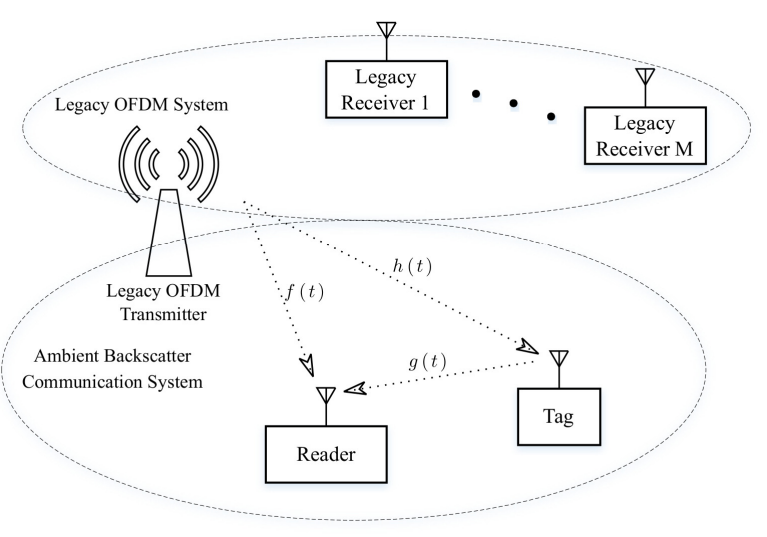

Fig. 1. The System Model

for communication and may be powered by an RF-energy harvester.

As shown in Fig. 1, let $h(t), f(t)$, and $g(t)$ denote, respectively, the bandpass impulse responses of the multipath Rayleigh fading channels between the legacy transmitter and the tag, the legacy transmitter and the reader, and the tag and the reader. The multi-path delay spreads corresponding to these channels are denoted, respectively, by $\tau_{h}, \tau_{f}$ and $\tau_{g}$. All channels are assumed to be mutually independent.

Denote the bandpass signal transmitted from the OFDM legacy transmitter during a symbol interval as

$$
s(t)=\Re\left\{\sqrt{p} s_{l}(t) \mathrm{e}^{j 2 \pi f_{c} t}\right\},
$$

where $p$ is the average transmitted power, $s_{l}(t)$ is the baseband representation of $s(t), f_{c}$ is the carrier frequency, and $\Re\{\cdot\}$ denotes the real-part operator. The received signal at the tag can be written as

$$
x(t)=\Re\left\{\left[\sqrt{p} s_{l}(t) * h_{l}(t)\right] \mathrm{e}^{j 2 \pi f_{c} t}\right\},
$$

where $*$ denote linear convolution, and $x_{l}(t)=\sqrt{p} s_{l}(t) *$ $h_{l}(t)$ is the baseband representation of $x(t)$.

The tag modulates its information onto the received signal by changing its antenna impedance. Let $b_{l}(t)$ denote the baseband representation of the tag's modulation waveform with corresponding bandpass signal $b(t)$. As widely assumed in the literature on ambient backscatter communications [8], [9], [11], we assume that no noise is added at the tag. This assumption arises from the fact that the tag has no active RF components. Thus, the signal backscattered from the tag will be $x(t) b(t)$.

The received signal at the reader can be written as

$$
y(t)=\underbrace{[x(t) b(t)] * g(t)}_{y^{b}(t)}+\underbrace{s(t) * f(t)}_{y^{d}(t)}+w(t),
$$

where $y^{b}(t)=[x(t) b(t)] * g(t)$ is the signal backscattered from the tag, $y^{d}(t)=\sqrt{p} s(t) * f(t)$ is the signal received directly from the legacy transmitter, and $w(t)$ is the bandpass "white" Gaussian noise random process, which is independent of both $y^{b}(t)$ and $y^{d}(t)$. Note that tag's information in present only in the term $y^{b}(t)$, while $y^{d}(t)$ is the direct-link 
(i.e. legacy-transmitter to reader) interference. The baseband representation of (3) can be written as

$$
y_{l}(t)=y_{l}^{b}(t)+y_{l}^{d}(t)+w_{l}(t),
$$

where $y_{l}^{b}(t), y_{l}^{d}(t)$, and $w_{l}(t)$ denote the baseband representations of $y^{b}(t), y^{d}(t)$, and $w(t)$, respectively.

At the reader, the received signal is down-converted to baseband and passed through an analog-to-digital converter (ADC). The resultant discrete-time baseband sequence, for one OFDM symbol, can be written as

$$
y_{l}[n]=y_{l}^{b}[n]+y_{l}^{d}[n]+w_{l}[n], \quad n=1,2, \ldots, N_{f}+N_{c p},
$$

where $N_{f}$ is the number of subcarriers, or equivalently the length of the fast Fourier transform (FFT), $N_{c p}$ is the cyclic prefix length, and $w_{l}[n]$ is complex baseband additive white Gaussian noise (AWGN) with zero-mean and variance $\sigma_{w}^{2}$. Let $h_{l}[n], f_{l}[n]$, and $g_{l}[n]$ denote the discrete-time baseband representation of $h(t), f(t)$, and $g(t)$, respectively. Hence, we can write $y_{l}^{b}[n]=\left(x_{l}[n] b_{l}[n]\right) * g_{l}[n]$ and $y_{l}^{d}[n]=$ $\sqrt{p} s_{l}[n] * f_{l}[n]$. The discrete-time channels' lengths are given by $L_{h}=\left\lfloor\tau_{h} f_{s}\right\rfloor, L_{f}=\left\lfloor\tau_{f} f_{s}\right\rfloor$, and $L_{g}=\left\lfloor\tau_{g} f_{s}\right\rfloor$, where $f_{s}$ is the sampling frequency. Let $\tau \triangleq \max \left\{\tau_{f}, \tau_{h}+\tau_{g}\right\}$ denote the maximum channel delay spread; hence, $L \triangleq$ $\max \left\{L_{f}, L_{h}+L_{g}-1\right\}$ denote the discrete-time length of maximum channel delay spread. Finally, it is reasonable to assume that $L_{g}=1$, since the distance between the tag and reader is fairly small in practice [11]. Hence, the backscattered signal at the reader can be simplified to $y_{l}^{b}[n]=g x_{l}[n] b_{l}[n]$. In the rest of the paper, we use the discrete-time baseband model and drop the subscript $l$ for notational convenience.

Our goal is to design the tag modulation waveform, $b[n]$, and the detector at the reader to be able to extract the tag information in $b[n]$ from the received signal $y[n]$ without knowing either the transmitted OFDM symbol $s[n]$ or the relevant channels $h[n], f[n]$, and $g$.

\section{Transceiver Design And Performance ANALYSIS}

In this section, we propose a modulation scheme for backscatter communications over ambient OFDM signals. We describe the tag modulation waveform and study the detector design. We also analyze the error performance of the proposed scheme and obtain exact expressions for the average error probability.

\section{A. Backscatter Waveform Design}

In OFDM systems, not all subcarriers are used to transmit information. The edge subcarriers are usually left null. For example, in the LTE standard, for the $5 \mathrm{MHz}$ channel bandwidth, the number of subcarriers is 512, out of which 211 are left null [13]. Let $\mathcal{U}$ and $\mathcal{D}$ denote the set of null subcarriers and the set of data subcarriers (including the DC subcarrier), respectively, and denote the cardinality of a set $\mathcal{X}$ by $|\mathcal{X}|$.

We exploit the structure of the OFDM symbol spectrum by designing the tag modulation waveform $b[n]$ to shift the backscattered energy into these null subcarriers so that a simple energy detector can be used to decode the tag information. This exempts the reader from knowing the transmitted OFDM symbol or any of the relevant channels. Similar to [11], [12], every backscatter symbol spans the duration of one legacy OFDM symbol. The tag uses the following waveform to convey one information bit per OFDM symbol,

$$
b[n] \triangleq \mathrm{e}^{i \pi B n}, \quad n=1,2, \ldots, N_{f}+N_{c p}
$$

where $B \in\{0,1\}$ is the information bit being transmitted. Hence, to transmit a ' 1 ' bit the tag will alternate its antenna impedance between two states, one state causes a phase shift of $\pi$ and the other state provides no phase shift, while to transmit a ' 0 ' bit the tag keeps its antenna impedance constant at a value that provides no phase shift. Using this tag waveform, the backscattered signal received at the reader can be written as

$$
y^{b}[n]=g x[n] \mathrm{e}^{i \pi B n} .
$$

Taking the discrete Fourier transform of (7), the backscattered signal spectrum can be written as

$$
Y^{b}[m]=g X[m] \circledast \delta\left[m-\frac{B f_{s}}{2}\right]=g X\left[m-\frac{B f_{s}}{2}\right],
$$

where $\circledast$ denotes circular convolution, and $X[\mathrm{~m}]$ is the discrete Fourier transform (DFT) of $x[n]$. Thus, from the viewpoint of the frequency domain, to transmit a ' 1 ' bit, the tag shifts the spectrum of the backscattered signal. This shift in frequency will cause a large fraction of the backscattered energy to fall into all the null subcarriers. Hence, an energy detector over the null subcarriers can be used at the receiver to decode the tag information.

Before we conclude this section, it is worth mentioning that this spectrum shifting scheme could be easily extended to allow higher order modulations; however, we are unable to present the details due to space limitations.

\section{B. Detector}

In this section, we design the detector for the modulation scheme introduced the previous subsection. The reader only knows the set of null subcarriers, $\mathcal{U}$, along the edges of the ambient OFDM symbol, and the average SNR, but has no knowledge of the OFDM ambient signal, $s[n]$, or the relevant channels $h[n], f[n]$, or $g$. Since the tag transmits its information by shifting the spectrum of the backscattered signal into the null subcarriers, an energy detector is used to collect the energy in the null subcarriers, and decode the tags information. However, not all null subcarriers may be used for energy detection, since many null subcarriers fall outside the channel bandwidth and maybe subject to adjacent channel interference.

Decision Statistic: Let $\mathcal{U}_{i} \in \mathcal{U}$ and $\mathcal{U}_{o} \in \mathcal{U}$ denote, respectively, the sets of in-band and out-of-band null subcarriers. At the reader, the cyclic prefix is discarded, and the remaining $N_{f}$ samples are passed through an FFT block. Let $Y[m]$ denote 


$$
p(z \mid B=1)=\sum_{j=1}^{J} \int_{0}^{\infty} \frac{2\left|\mathcal{U}_{i}\right|}{\lambda_{j} \bar{\gamma}}\left(\prod_{k \neq j} \frac{\frac{1}{\lambda_{k}}}{\frac{1}{\lambda_{k}}-\frac{1}{\lambda_{j}}}\right) \mathrm{e}^{-\frac{z+2\left|\mathcal{U}_{i}\right| \gamma}{2}}\left(\frac{z}{2\left|\mathcal{U}_{i}\right| \gamma}\right)^{\frac{\left|\mathcal{U}_{i}\right|-1}{2}} \mathbf{I}_{\left|\mathcal{U}_{i}\right|-1}\left(\sqrt{2\left|\mathcal{U}_{i}\right| \gamma z}\right) \mathbf{K}_{0}\left(2 \sqrt{\frac{\left|\mathcal{U}_{i}\right| \gamma}{\lambda_{j} \bar{\gamma}}}\right) \mathrm{d} \gamma,
$$

the output of the FFT. Hence, the test statistic can be written as

$$
z=\frac{2}{\sigma_{w}^{2}} \sum_{m \in \mathcal{U}_{i}}|Y[m]|^{2} .
$$

Under $\mathcal{H}_{0}$, the hypothesis that the tag transmitted a ' 0 ' bit, the null subcarriers contain only noise ${ }^{1}$ and $z$ is the sum of the squares of $2\left|\mathcal{U}_{i}\right|$ standard Gaussian random variables. Hence, $p(z \mid B=0)$ is a central Chi-squared distribution with $2\left|\mathcal{U}_{i}\right|$ degrees of freedom [14]. On the other hand, when the tag is transmitting a ' 1 ' bit, the distribution of the decision statistic $z$ is fairly complicated. Under $\mathcal{H}_{1}$, the hypothesis that the transmitted bit is ' 1 ', the received energy in the null subcarriers depends on the random channels $g$ and $h[n]$. Therefore, the instantaneous detection SNR is a random variable and can be written as

$$
\gamma=\frac{p|g|^{2} \sum_{m \in \mathcal{U}_{i}}|H[m]|^{2}}{\left|\mathcal{U}_{i}\right| \sigma_{w}^{2}},
$$

where $\{H[m]\}_{m \in \mathcal{U}_{i}}$ are the flat-fading channel coefficients seen by the in-band null-subcarriers. However, conditional on the instantaneous received SNR, $\gamma$, the decision statistic distribution $p(z \mid \gamma, B=1)$ is a non-central Chi-squared with $2\left|\mathcal{U}_{i}\right|$ degrees of freedom, and non-centrality parameter $\Lambda=2\left|\mathcal{U}_{i}\right| \gamma$ [14]. Hence, the distribution of the decision statistic under $\mathcal{H}_{1}$ could be found by averaging over the distribution of the instantaneous SNR. The instantaneous SNR, $\gamma$, is a scaled product of two random variables: $|g|^{2}$, which is an exponential random variable, and $q \triangleq \sum_{m \in \mathcal{U}_{i}}|H[m]|^{2}$, which is the sum of $\left|\mathcal{U}_{i}\right|$ correlated exponential random variables. The correlation arises from the fact that the subcarrier spacing has to be smaller than the coherence bandwidth. Let $\mathbf{h}$ denote the vector comprising the channel coeficients $\{H[m]\}_{m \in \mathcal{U}_{i}}$. Then, using the technique in [15], the distribution of $q$ can be found to be

$$
f(q)=\sum_{j=1}^{J}\left(\prod_{k \neq j} \frac{\frac{1}{\lambda_{k}}}{\frac{1}{\lambda_{k}}-\frac{1}{\lambda_{j}}}\right) \frac{1}{\lambda_{j}} \mathrm{e}^{-\frac{q}{\lambda_{j}}},
$$

where $\left\{\lambda_{i}\right\}_{j=1}^{J}$ are the non-zero eigenvalues of the co-variance matrix $\mathbf{R}_{H}=E\left[\mathbf{h h}^{\dagger}\right]$. Hence, the instantaneous SNR distribution can be readily found, using the product distribution formula, to be

$$
f(\gamma)=\sum_{j=1}^{J}\left(\prod_{k \neq j} \frac{\frac{1}{\lambda_{k}}}{\frac{1}{\lambda_{k}}-\frac{1}{\lambda_{j}}}\right) \frac{2\left|\mathcal{U}_{i}\right|}{\lambda_{j} \bar{\gamma}} \mathbf{K}_{0}\left(2 \sqrt{\frac{\left|\mathcal{U}_{i}\right| \gamma}{\lambda_{j} \bar{\gamma}}}\right),
$$

${ }^{1} \mathrm{We}$ assume ICI is negligable since (1) there is no doppler spread since the tag, reader and legacy transmitter are stationary. (2) at practical low-medium SNRs, noise is dominant. (3) ICI is weakest at edge subcarriers. where $\bar{\gamma} \triangleq E[\gamma]$ is the average detection SNR and $\mathbf{K}_{m}(\cdot)$ is the modified Bessel function of the second kind and $m$-th order. Alternatively, we can write the SNR distribution as [16]

$$
f(\gamma)=\sum_{j=1}^{J}\left(\prod_{k \neq j} \frac{\frac{1}{\lambda_{k}}}{\frac{1}{\lambda_{k}}-\frac{1}{\lambda_{j}}}\right) \frac{1}{\gamma} G_{0,2}^{2,0}\left(\overline{1,1} \mid \frac{\left|\mathcal{U}_{i}\right| \gamma}{\lambda_{j} \bar{\gamma}}\right)
$$

where $G ., .(: \mid \cdot)$ is the Meijer G-function. The expression in (13) will be useful later on in computing the average probability of error. Using (12) and the expression for the probability distribution function (pdf) of the noncentral Chisquared distribution, the distribution of the decision statistic under $\mathcal{H}_{1}$ can be computed from (14), where $\mathbf{I}_{m}(\cdot)$ is the modified Bessel function of the first kind and $m$-th order.

The integral in (14) can be solved by expressing the exponential function and the two Bessel functions in terms of the Meijer-G function then applying (07.34.21.0081.01) from [16] to yield

$$
\begin{aligned}
& p(z \mid B=1)=\sum_{j=1}^{J} \pi\left(\prod_{k \neq j} \frac{\frac{1}{\lambda_{k}}}{\frac{1}{\lambda_{k}}-\frac{1}{\lambda_{j}}}\right)\left(\frac{z}{2}\right)^{\left|\mathcal{U}_{i}\right|-1} \mathrm{e}^{-\frac{z}{2}} \\
& \times G_{1,0: 1,3: 0,2}^{1,0: 1,0: 2,0}\left(0 \mid \begin{array}{c|c|c}
- & \frac{1}{2} & 1,1 \\
0,1 & -\left|\mathcal{U}_{i}\right|, \frac{1}{2} & \frac{1}{\lambda_{j} \bar{\gamma}}, \frac{z}{2}
\end{array}\right) .
\end{aligned}
$$

Note that this proposed scheme eliminates direct link interference at the reader as the energy from the direct link exists only on the data subcarriers.

Error Performance: Next, we analyze the probability of error for the proposed scheme. Let $\delta$ denote the decision threshold. Then, since the tag transmitted bits are equally probable to be ones or zeros, the average probability of error is given by

$$
P_{e}(\delta)=\frac{1}{2} P_{e \mid B=0}(\delta)+\frac{1}{2} P_{e \mid B=1}(\delta) .
$$

Both $P_{e \mid B=0}(\delta)=\operatorname{Pr}(\hat{B}=1 \mid B=0)$ and $P_{e \mid B=1}(\delta)=$ $\operatorname{Pr}(\hat{B}=0 \mid B=1)$ are functions of the decision threshold $\delta$, which should be chosen to minimize the average probability of error $P_{e}(\delta) . P_{e \mid B=0}(\delta)$ is independent of the SNR and is given by the tail probability of the central Chi-squared distribution as [17]

$$
P_{e \mid B=0}(\delta)=\frac{\Gamma\left(\left|\mathcal{U}_{i}\right|, \frac{\delta}{2}\right)}{\Gamma\left(\left|\mathcal{U}_{i}\right|\right)}
$$

where $\Gamma(s, x)=\int_{x}^{\infty} t^{s-1} \mathrm{e}^{-t} \mathrm{~d} t$ is the upper incomplete Gamma function, and $\Gamma(s)=\int_{0}^{\infty} t^{s-1} \mathrm{e}^{-t} \mathrm{~d} t$ is the "complete" Gamma function. Whereas, $P_{e \mid B=1}(\delta)$ is dependent on the instantaneous SNR and subsequently on the random 
backscatter channel. We can write $P_{e \mid B=1}(\delta)$, conditional on the instantaneous SNR, using the cumulative distribution function $(\mathrm{CDF})$ of the noncentral Chi-squared random variable as

$$
P_{e \mid \gamma, B=1}(\delta)=1-Q_{\left|\mathcal{U}_{i}\right|}\left(\sqrt{2\left|\mathcal{U}_{i}\right| \gamma}, \sqrt{\delta}\right),
$$

where $Q .(\cdot, \cdot)$ is the Marcum Q-Function. Thus, using (13), we can average $P_{e \mid \gamma, B=1}(\delta)$ over the distribution of the instantaneous SNR to obtain (19).

$$
\begin{aligned}
P_{e \mid B=1}(\delta) & =1-\sum_{j=1}^{J}\left(\prod_{k \neq j} \frac{\frac{1}{\lambda_{k}}}{\frac{1}{\lambda_{k}}-\frac{1}{\lambda_{j}}}\right) \int_{0}^{\infty} \gamma^{-1} \\
& \times Q_{\left|\mathcal{U}_{i}\right|}\left(\sqrt{2\left|\mathcal{U}_{i}\right| \gamma}, \sqrt{\delta}\right) G_{0,2}^{2,0}\left(\overline{1,1} \mid \frac{\left|\mathcal{U}_{i}\right| \gamma}{\lambda_{j} \bar{\gamma}}\right) \mathrm{d} \gamma
\end{aligned}
$$

Using Theorem 1 in [18], the integral in (19) can be evaluated in terms of the bivariate Meijer G-function [19]. Hence, we can write the average probability of error as a function of the threshold as in (20)

$$
\begin{aligned}
& P_{e}(\delta)=\frac{1}{2}+\frac{\Gamma\left(\left|\mathcal{U}_{i}\right|, \frac{\delta}{2}\right)}{2 \Gamma\left(\left|\mathcal{U}_{i}\right|\right)}+\sum_{j=1}^{J} \frac{1}{2 \lambda_{j} \bar{\gamma}}\left(\prod_{k \neq j} \frac{\frac{1}{\lambda_{k}}}{\frac{1}{\lambda_{k}}-\frac{1}{\lambda_{j}}}\right) \\
& \left.\quad \times G_{1,0: 1,3: 1,3}^{1,0: 1,0: 2,(} \mid \begin{array}{c|c|c|c}
0 & \frac{1}{2} & 1 & \frac{\sqrt{\delta}}{2}, \frac{1}{\lambda_{j} \bar{\gamma}}
\end{array}\right) .
\end{aligned}
$$

The bivariate Meijer G-function is not available as a built-in function in well-known computational software packages (e.g. MATLAB, Wolfram Mathematica, Maple); however, there exist two implementations in the literature, one using Wolfram Mathematica in [20], and another one using MATLAB in [21].

Detector Threshold: The decision threshold should be chosen to minimize the average probability of error in (20), i.e.

$$
\delta^{*}=\arg \min _{\delta} P_{e}(\delta)
$$

Since the tag transmits ones and zeros with equal probability, the optimal decision rule that minimizes the probability of error is the maximum likelihood (ML) rule given by

$$
\hat{B}= \begin{cases}1, & p(z \mid B=1) \geq p(z \mid B=0), \\ 0, & p(z \mid B=0)>p(z \mid B=1) .\end{cases}
$$

Hence, the optimal decision threshold, $\delta^{*}$, lies at the intersection of the two likelihood functions, $p(z \mid B=0)$ and $p(z \mid B=1)$. Unfortunately, a closed-form expression for $\delta^{*}$ cannot be found analytically. However, it can be easily found numerically, for any SNR, using a simple, one-dimensional line search.

\section{Results And Discussion}

In this section, we present simulation results to evaluate the performance of the proposed backscatter modulation scheme and verify the analysis in Section III. We study the effects of the maximum channel delay spread, $\tau$, and the OFDM symbol size, $N_{f}$, on the error performance. Suppose the ambient OFDM signal is a $10 \mathrm{MHz}$ LTE carrier [13]; hence, the FFT size is $N_{f}=1024$, the "normal" CP length is $N_{c p}=72$, and the number of null subcarriers is $|\mathcal{U}|=423$ of which $\left|\mathcal{U}_{i}\right|=64$ are in-band. The maximum channel delay spread, $\tau$, is specified in each figure. We use the scheme in [11] as a baseline for comparison.

The scheme in [11] takes advantage of the fact that the portion of the cyclic prefix not affected by the multipath channel, i.e. $n=L, \ldots, N_{c p}$, is repeated in the received signal. The tag waveform is designed to either change its antenna impedance to phase-shift the ambient signal by $\pi$ for the second part of the OFDM symbol to transmit a ' 1 ' bit or keep the phase unchanged to transmit a ' 0 ' bit. Let $B$ denote the tag transmitted bit; hence, for $n=L, \ldots, N_{c p}$, we have

$$
r[n] \triangleq y[n]-y\left[n+N_{f}\right]= \begin{cases}u[n]+v[n], & B=1, \\ v[n], & B=0,\end{cases}
$$

where $y[n]$ is the received signal at the reader, $u[n]=$ $2 g \sqrt{p} \sum_{l=1}^{L h} s[n-l] h[l]$, and $v[n]=w[n]-w\left[n+N_{f}\right]$. Hence, an energy detector can be used to decode the tag information using the test statistic $\frac{1}{\sigma_{w}^{2}} \sum_{L}^{N_{c p}}|r[n]|^{2}$. Note that the modulation scheme in [11] necessitates that the reader estimate the maximum channel delay spread length $L$, and if this delay spread is equal to the cyclic prefix, the scheme fails. Our proposed scheme does not suffer from this limitation.

Fig. 2 compares the average error performance of the proposed scheme to the scheme in [11] for different values of maximum channel delay spread, $\tau$. As expected, the performance of the baseline scheme in [11] deteriorates rapidly as the maximum channel delay spread increases since the usable part of the cyclic prefix diminishes. On the other hand, the proposed scheme is hardly affected by maximum channel delay spread. Actually, the performance of the proposed scheme slightly improves with increasing delay spread, as the coherence bandwidth decreases and the channel coefficients for the null subcarriers become less correlated. For a delay spread of $4 \mu \mathrm{s}$, the proposed scheme outperforms the baseline scheme by almost $4 \mathrm{~dB}$ at an error rate of $10^{-2}$. We also notice that the probability of error obtained by Monte-Carlo simulations coincides with the analytical probability of error, which verifies our analysis.

Finally, in Fig. 3, we study the effects of varying the ambient OFDM symbol size on the error performance of the proposed scheme and compare it with the baseline scheme in [11]. We use the LTE OFDM symbol parameters for the $5 \mathrm{MHz}, 10 \mathrm{MHz}$ and $20 \mathrm{MHz}$ channel bandwidths [13]. We assume the maximum channel delay spread, $\tau$, is $3 \mu \mathrm{s}$, which is a typical value in urban outdoor environments. Simulation results show that increasing the OFDM symbol size improves the performance of both the proposed and baseline schemes; however, the proposed scheme benefits more from increasing the OFDM symbol size. Moreover, for the used typical value of delay spread, the proposed scheme outperforms the baseline scheme for all three OFDM symbol sizes. 


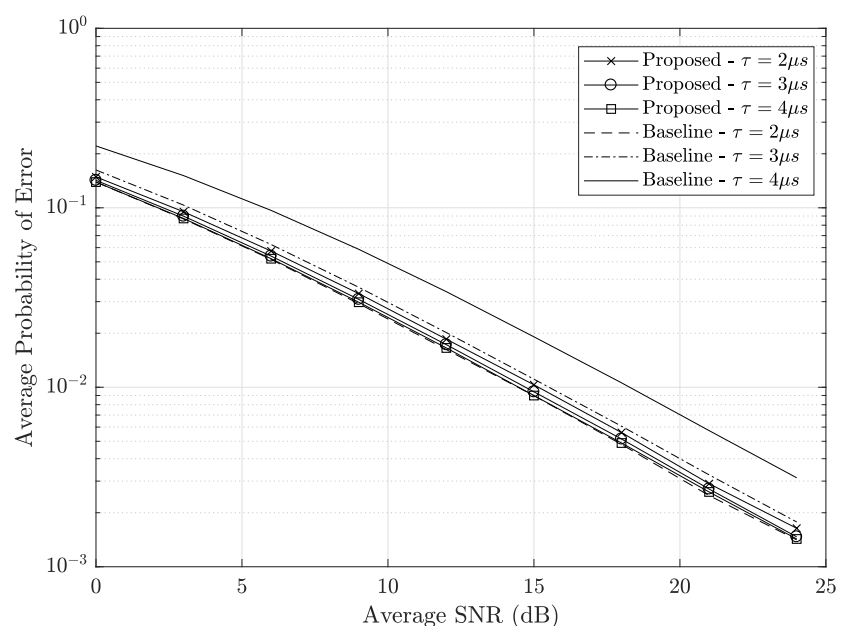

Fig. 2. Average probability of error for different values of maximum channel delay spread. Lines correspond to Monte-Carlo simulations and markers correspond to analytical expressions. Baseline scheme from [11]. $N_{f}=1024, N_{c p}=72,\left|\mathcal{U}_{i}\right|=64, L_{g}=1$.

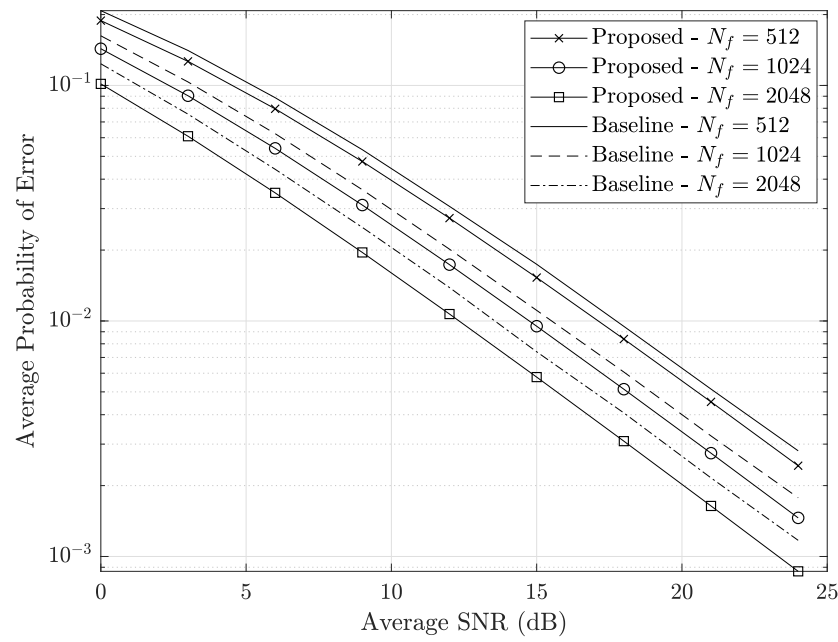

Fig. 3. Average probability of error for different OFDM symbol sizes, and a maximum channel delay spread of $3 \mu \mathrm{s}$. Lines correspond to MonteCarlo simulations and markers correspond to analytical expressions. Baseline scheme from [11]. $L_{g}=1$.

\section{CONCLUSION}

We introduced a novel backscatter modulation technique over ambient OFDM signals. In particular, we took advantage of the null subcarriers found in all OFDM signals and designed the tag modulation waveform such that the backscattered energy lie mostly in the null subcarriers. Hence, a simple energy detector can be used to detect the backscattered information without requiring knowledge of the ambient OFDM symbol or the relevant channels. This scheme avoids direct link interference since there is no energy from the ambient transmission in the null subcarriers, and could be extended to allow higher order modulation. We have also analyzed the error performance of the proposed scheme and provided an exact expression for the error probability in terms of the bivariate Meijer G-function. Simulation results corroborated our analysis and showed that the proposed scheme outperforms other schemes available in the literature for ambient backscatter over ambient OFDM signals in different scenarios.

\section{REFERENCES}

[1] C. Boyer and S. Roy, "Backscatter communication and RFID: coding, energy, and MIMO analysis," IEEE Trans. Commun., vol. 62, no. 3 , pp. 770-785, Mar. 2014.

[2] V. Liu, A. Parks et al., "Ambient backscatter: wireless communication out of thin air," in Proc. of the ACM SIGCOMM Conf., Hong Kong, China, Aug. 2013, pp. 39-50.

[3] B. Kellogg, A. Parks et al., "Wi-fi backscatter: internet connectivity for RF-powered devices," in Proc. of the ACM SIGCOMM Conf., Chicago, IL, Aug. 2014, pp. 607-618.

[4] A. N. Parks, A. Liu et al., "Turbocharging ambient backscatter communication," in Proc. of the ACM SIGCOMM Conf., Chicago, IL, Aug. 2014, pp. 619-630.

[5] D. Bharadia, K. R. Joshi et al., "BackFi: high throughput WiFi backscatter," in Proc. of the ACM SIGCOMM Conf., London, U.K., Aug. 2015, pp. 283-296.

[6] V. Iyer, V. Talla et al., "Inter-technology backscatter: towards internet connectivity for implanted devices," in Proc. of the ACM SIGCOMM Conf., Florianópolis, Brazil, Aug. 2016, pp. 356-369.

[7] R. Duan, R. Jäntti et al., "On the achievable rate of bi-static modulated re-scatter systems," IEEE Trans. Veh. Technol., vol. 66, no. 10, pp. $9609-9613$, Oct. 2017.

[8] G. Wang, F. Gao et al., "Uplink detection and BER analysis for ambient backscatter communication systems," in Proc. IEEE Global Commun. Conf. (GLOBECOM), San Diego, CA, Dec. 2015.

[9] Z. Mat, T. Zeng et al., "Signal detection for ambient backscatter system with multiple receiving antennas," in Proc. IEEE 14th Canadian Workshop on Inform. Theory (CWIT), St. John's, NL, Canada, Jul. 2015 , pp. 50-53.

[10] J. Qian, F. Gao, and G. Wang, "Signal detection of ambient backscatter system with differential modulation," in Proc. IEEE Int. Conf. on Acoust., Speech and Signal Process. (ICASSP), Shanghai, China, Mar. 2016, pp. 3831-3835.

[11] G. Yang and Y. C. Liang, "Backscatter communications over ambient OFDM signals: transceiver design and performance analysis," in Proc. IEEE Global Commun. Conf. (GLOBECOM), Washington, DC, Dec. 2016.

[12] D. Darsena, G. Gelli, and F. Verde, "Modeling and performance analysis of wireless networks with ambient backscatter devices," IEEE Trans. Commun., vol. 65, no. 4, pp. 1797-1814, Apr. 2017.

[13] A. Ghosh, J. Zhang et al., Fundamentals of LTE, 1st ed. Upper Saddle River, NJ: Prentice Hall Press, 2010.

[14] H. Urkowitz, "Energy detection of unknown deterministic signals," Proc. of the IEEE, vol. 55, no. 4, pp. 523-531, Apr. 1967.

[15] I. S. Ansari, F. Yilmaz et al., "On the sum of gamma random variates with application to the performance of maximal ratio combining over nakagami-m fading channels," in IEEE 13th Int. Workshop on Signal Process. Advances in Wireless Commun. (SPAWC), Cesme, Turkey, Jun. 2012, pp. 394-398.

[16] Wolfram Research Inc., The Wolfram Functions Site. [Online]. Available: http://functions.wolfram.com

[17] F. F. Digham, M. S. Alouini, and M. K. Simon, "On the energy detection of unknown signals over fading channels," in Proc. IEEE Int. Conf. on Commun., vol. 5, May 2003, pp. 3575-3579.

[18] P. C. Sofotasios, L. Mohjazi et al., "Energy detection of unknown signals over cascaded fading channels," IEEE Antennas Wireless Propag. Lett., vol. 15, pp. 135-138, 2016.

[19] B. L. Sharma, "On the generalized function of two variables," Annales de Soc. Sci. de Bruxelles, no. 79, pp. 26-40, 1965.

[20] I. S. Ansari, S. Al-Ahmadi et al., "A new formula for the BER of binary modulations with dual-branch selection over generalized-K composite fading channels," IEEE Trans. Commun., vol. 59, no. 10, pp. 26542658, Oct. 2011.

[21] H. Chergui, M. Benjillali, and S. Saoudi, "Performance analysis of project-and-forward relaying in mixed MIMO-pinhole and Rayleigh dual-hop channel," IEEE Commun. Lett., vol. 20, no. 3, pp. 610-613, Mar. 2016. 